\title{
The impact of breast cancer biological subtyping on tumor size assessment by ultrasound and mammography - a
} retrospective multicenter cohort study of 6543 primary breast cancer patients

\author{
Roland Gregor Stein ${ }^{*}$, Daniel Wollschläger ${ }^{2}$, Rolf Kreienberg ${ }^{3}$, Wolfgang Janni ${ }^{3}$, Manfred Wischnewsky ${ }^{4}$,
} Joachim Diessner ${ }^{1}$, Tanja Stüber ${ }^{1}$, Catharina Bartmann ${ }^{1}$, Mathias Krockenberger', Jörg Wischhusen', Achim Wöckel ${ }^{1}$, Maria Blettner ${ }^{2}$, Lukas Schwentner ${ }^{3}$ and the BRENDA Study Group

\begin{abstract}
Background: Mammography and ultrasound are the gold standard imaging techniques for preoperative assessment and for monitoring the efficacy of neoadjuvant chemotherapy in breast cancer. Maximum accuracy in predicting pathological tumor size non-invasively is critical for individualized therapy and surgical planning. We therefore aimed to assess the accuracy of tumor size measurement by ultrasound and mammography in a multicentered health services research study.

Methods: We retrospectively analyzed data from 6543 patients with unifocal, unilateral primary breast cancer. The maximum tumor diameter was measured by ultrasound and/or mammographic imaging. All measurements were compared to final tumor diameter determined by postoperative histopathological examination. We compared the precision of each imaging method across different patient subgroups as well as the method-specific accuracy in each patient subgroup.
\end{abstract}

Results: Overall, the correlation with histology was 0.61 for mammography and 0.60 for ultrasound. Both correlations were higher in pT2 cancers than in pT1 and pT3. Ultrasound as well as mammography revealed a significantly higher correlation with histology in invasive ductal compared to lobular cancers $(p<0.01)$. For invasive lobular cancers, the mammography showed better correlation with histology than ultrasound $(p=0.01)$, whereas there was no such advantage for invasive ductal cancers. Ultrasound was significantly superior for HR negative cancers $(p<0.001)$. HER2/neu positive cancers were also more precisely assessed by ultrasound $(p<0.001)$. The size of HER2/neu negative cancers could be more accurately predicted by mammography $(p<0.001)$.

Conclusion: This multicentered health services research approach demonstrates that predicting tumor size by mammography and ultrasound provides accurate results. Biological tumor features do, however, affect the diagnostic precision.

Keywords: Breast cancer, Ultrasound, Mammography, Tumor size, Histopathology

\footnotetext{
* Correspondence: stein_r@ukw.de

'Department of Obstetrics and Gynecology, Würzburg University Hospital,

Josef-Schneider-Str. 4, 97080 Würzburg, Germany

Full list of author information is available at the end of the article
} 


\section{Background}

Breast cancer remains the most common malignancy among women with an incidence of about 70,000 cases per year in Germany (http://www.krebsgesellschaft.de/basisinformationen-krebs/krebsarten/brustkrebs.html). Distinct biological subgroups of breast cancer show significantly different tumor growth and prognosis as well as therapeutic options [1]. The invasive carcinoma of no special type (NST), also known as invasive ductal carcinoma or ductal carcinoma NOS (not otherwise specified), accounts for about $70-80 \%$ of breast cancers. Less common are invasive lobular cancers with $10-15 \%$ of all breast cancers and rare subtypes such as medullary, tubular or mucinous carcinoma [2]. Using cDNA microarray analysis, Perou et al. defined different biological subgroups of breast cancers with impact on tumor biology and clinical appearance [1]: Luminal A and B breast cancers as well as HER2/neu positive and basal like breast cancer. Gene expression profiling is not yet part of routine tumor analysis But hormone receptor expression, HER2/neu overexpression and proliferation markers represent surrogate markers for biological breast cancer subgroups.

Tumor resection is still essential for therapy concepts in breast cancer care. In many cases, breast-conserving surgery can be performed instead of mastectomy. Incomplete or marginal tumor resection requires a reresection. Imaging technologies are thus essential not only for diagnosis but also for preoperative assessment of breast cancer. Especially for non-palpable tumors, imaging plays an outstanding role.

Previous studies showed that mammography slightly overestimates tumor size, whereas ultrasound tends to underestimate tumor size [3]. Other groups found ultrasound to provide the more exact estimates for tumor size [4]. In these studies, there was no separate evaluation for the different biological subgroups of breast cancer. A single-center retrospective study of 121 patients [5] found that ultrasound-based assessments tend to underestimate in particular the size of invasive ductal cancer with ductal carcinoma in situ and invasive lobular as well as invasive ductal cancers. Bosch et al. published a prospective study that found ultrasound to be the best predictor of histological tumor size compared to mammography and physical examination. As ultrasound underestimated the tumor size, they suggested a formula for calculating the probable histological tumor size: Sonographic tumor size $(\mathrm{mm})+3 \mathrm{~mm}$ [6]. Ultrasound seems to be especially good in the assessment of tumors with less than $30 \mathrm{~mm}$ diameter [7]. Ramirez and colleagues found good correlations between ultrasound, mammography and especially MRI with histological tumor size [8].

According to German guidelines for breast cancer diagnostics and treatment, mammography is the standard imaging tool [9]. In case of high breast density (ACR
3-4), an ultrasound examination should be added to achieve higher sensitivity [10]. Both mammography and ultrasound are standard diagnostic tools for breast cancer assessment $[11,12]$. The role of magnetic resonance imaging (MRI) of the breast as preoperative assessment is controversial: In a metaanalysis of 9 clinical studies, Houssami and colleagues found that MRI did not reduce re-excisions but significantly increased the rate of modified radical mastectomies (MRM) [13, 14]. They suggest that a routine MRI in breast cancer patients could do more harm than good [13]. Though preoperative bilateral breast MRI could reduce the risk of a contralateral cancer recurrence, $\mathrm{Yi}$ et al. could not find any difference in local-regional recurrence rates [15].

The role of MRI in breast cancer imaging is still controversial while ultrasound and mammography remain the gold standard in care. We therefore aimed to investigate accuracy of the gold standard imaging techniques in a multicenter health services research approach investigating breast cancer imaging in a large daily routine cohort of patients.

\section{Methods}

We retrospectively analyzed data from 6543 breast cancer patients who were part of the BRENDA I study population. Patients with unifocal, unilateral primary breast cancer were included in the BRENDA I study. Data were collected from 1992 until 2008 at Ulm University Hospital and from 2002 until 2008 in 16 associated German breast cancer centers certified by the German Cancer Society. The study protocol was approved by the Ethics committee of the University of Ulm. Patients gave informed consent. Data regarding maximum tumor diameter in preoperative ultrasound, mammography, as well as histological tumor diameter were collected.

Patients were excluded from the analysis if they received neoadjuvant chemotherapy, if they suffered from bilateral, multicenter or inflammatory breast cancer as well as non-invasive tumors. In case of missing diagnostic data, the patients were also excluded.

The maximum tumor diameter was measured by imaging as well as by pathologic examination. In case of follow-up resections, tumor diameters were added excluding ductal carcinoma in situ (DCIS) and lobular carcinoma in situ (LCIS).

Endocrine responsiveness was categorized according to the 2007 St. Gallen Consensus Criteria [16].

\section{Statistical analysis}

Statistical analysis was performed using $\mathrm{R}$ (version 3.1 [17]). Patient characteristics were described with percentages, mean values and standard deviations. Precision (variability) and accuracy (systematic bias) of imaging methods were analyzed separately. Precision of mammography and 
ultrasound tumor size measurements were assessed by calculating Pearson's correlation coefficient with histological tumor size. T-tests were used to compare the independent correlation coefficients of the same imaging method between patient groups. To compare the correlation coefficients between imaging methods for the same patient group, Williams' test for the difference between two dependent correlations sharing one variable (histological tumor size) was used. Accuracy of imaging methods was assessed by their respective mean differences to histology measurements. Numerical results were complemented by visual evaluation of Bland-Altman plots that show the difference between the tumor diameter as measured by two methods against the mean of both measurements.

To provide a detailed evaluation of precision of tumor size measurements by mammography as well as ultrasound with respect to histology, we performed several types of comparisons: A) Comparisons of each imaging method across different patient groups. B) Comparison between mammography and ultrasound within one patient group. Patient groups were defined by either their age, or by different tumor characteristics like histological sub-type. We finally compared the precision of the detection of a $20 \mathrm{~mm}$ tumor diameter cutoff (C.). The impact of patient age on imaging was analyzed, respectively (D.).

\section{Results}

\section{Description of the study population}

Six thousand five hundred forty-three patients were eligible for the study. The mean age at diagnosis was 61.9 (SD 13.0 years). Three thousand eight hundred fifty-nine patients were stage pT1, 2469 with pT2 and 217 patients with pT3. Four thousand two hundred ten patients $(64.3 \%)$ showed pN0 status. $10.8 \%$ of the tumors were graded as G1, the majority of tumors were G2 $(61.8 \%)$ and $27.3 \%$ were G3 carcinomas. $14.4 \%$ of the tumors were hormone receptor (HR) negative. $14.8 \%$ of the tumors overexpressed HER2.

\section{Comparisons of each imaging method across different patient groups}

Mean difference between sonographic and histological tumor size The distributions of measured tumor size were generally unimodal and slightly right-skewed. The mean tumor diameter determined by ultrasound was $18.3 \mathrm{~mm}$ (SD $9.6 \mathrm{~mm}$ ), whereas the histological mean tumor diameter was $20.8 \mathrm{~mm}$ (SD $12.3 \mathrm{~mm}$ ). Data are summarized in Table 1. A Bland-Altman plot (Fig. 1a) indicates that measurement differences were proportional to tumor size with invasive lobular tumors being over-represented among tumors that are underestimated by ultrasound: Among 198 tumors underestimated by more than $20 \mathrm{~mm}, 68$ (34 \%) were invasive lobular cancers. Among 62 tumors overestimated by ultrasound by more than $20 \mathrm{~mm}$, only 4 (6\%) were invasive lobular cancers. Among 5642 tumors neither over- nor underestimated by more than $20 \mathrm{~mm}, 665$ (12\%) were invasive lobular cancers $(p<0.001)$.

Overall, ultrasound underestimated the histological tumor size with a mean difference of $2.5 \mathrm{~mm}$. This result also appeared in HR positive and HR negative tumors as well as in invasive ductal and invasive lobular cancers. There was a tendency towards decreasing sonographic accuracy in G3 high grade cancers.

Ultrasound accuracy was strongly dependent on tumor size: In pT1 cancers, the sonographic tumor diameter was higher than the histological tumor diameter. pT2 and pT3 cancers always had larger histological tumor diameters than determined by ultrasound.

Mean difference between mammographic and histological tumor size The overall mean histological diameter for patients examined by mammography was $21.0 \mathrm{~mm}$, and the mean mammographic diameter was $20.4 \mathrm{~mm}$. An overview of the mammography data is shown in Table 2. A Bland-Altman plot (Fig. 1b) indicates that measurement differences were proportional to tumor size with invasive lobular tumors being overrepresented among tumors that are underestimated by mammography: Among 110 tumors underestimated by more than $20 \mathrm{~mm}, 28$ (25\%) were invasive lobular cancers. Among 110 tumors overestimated by mammography by more than $20 \mathrm{~mm}$, only 12 (11 \%) were invasive lobular tumors. Among 4010 tumors neither over- nor underestimated by more than $20 \mathrm{~mm}, 434$ $(11 \%)$ were invasive lobular cancers $(p<0.001)$.

In both invasive ductal and invasive lobular cancer size was overall underestimated by mammography.

For mammography, tumor size was an important factor for the observed accuracy. pT1 cancers with a mean histologic diameter of $13.5 \mathrm{~mm}$ were overestimated in mammography while the opposite was true for pT2 and pT3. The difference peaked in the pT3 group with a mean histologic diameter of $62.6 \mathrm{~mm}$ and a mean difference of $18.3 \mathrm{~mm}$. Similarly, G1 cancers with a mean histological tumor diameter of $15.0 \mathrm{~mm}$ appeared larger in mammography whereas the size of G2 and G3 cancers was underestimated. Again, the peak mean difference was found in G3 cancers.

\section{Comparison between mammography and ultrasound within one patient group}

The correlation coefficients between histology, ultrasound and mammography for the respective subgroups are shown in Table 3. 
Table 1 Comparison of ultrasound and histology

\begin{tabular}{|c|c|c|c|c|c|c|c|}
\hline & $\begin{array}{l}\text { Mean histologic } \\
\text { diameter }\end{array}$ & $\begin{array}{l}\text { Mean } \\
\text { sonographic } \\
\text { diameter }\end{array}$ & $\begin{array}{l}\text { Mean } \\
\text { difference }\end{array}$ & $\begin{array}{l}\text { Mean relative } \\
\text { difference } \\
\text { (\% sonogaphic } \\
\text { tumor diameter) }\end{array}$ & $\begin{array}{l}\text { SD Histologic } \\
\text { diameter }\end{array}$ & $\begin{array}{l}\text { SD Sonographic } \\
\text { diameter }\end{array}$ & N \\
\hline Overall & 20.78 & 18.25 & 2.534 & 21.08 & 12.29 & 9.629 & 5902 \\
\hline Endocrine non-responsive & 23.77 & 21.10 & 2.666 & 17.24 & 13.98 & 10.205 & 848 \\
\hline Incomplete endocrine responsive & 19.88 & 18.18 & 1.702 & 16.91 & 11.60 & 10.408 & 1224 \\
\hline Highly endocine responsive & 20.43 & 17.62 & 2.811 & 23.44 & 12.00 & 9.078 & 3817 \\
\hline Not applicable & 14.69 & 23.46 & -8.769 & -29.05 & 12.85 & 16.179 & 13 \\
\hline Ductal invasive & 20.41 & 18.31 & 2.097 & 18.28 & 11.69 & 9.663 & 4257 \\
\hline Lobular invasive & 23.42 & 17.72 & 5.699 & 43.13 & 14.97 & 9.627 & 737 \\
\hline G1 & 15.03 & 14.48 & 0.5521 & 11.15 & 9.824 & 9.191 & 652 \\
\hline G2 & 20.08 & 17.61 & 2.4675 & 21.97 & 11.678 & 9.317 & 3636 \\
\hline G3 & 24.69 & 21.21 & 3.4854 & 23.08 & 13.289 & 9.697 & 1614 \\
\hline pT1 & 13.52 & 14.03 & -0.5067 & 6.024 & 5.159 & 7.066 & 3450 \\
\hline pT2 & 28.45 & 23.34 & 5.1141 & 35.658 & 6.989 & 8.347 & 2265 \\
\hline pT3 & 61.84 & 34.45 & 27.3904 & 122.220 & 17.761 & 16.055 & 187 \\
\hline Age $<50$ years & 20.06 & 17.70 & 2.353 & 21.99 & 12.40 & 9.127 & 1295 \\
\hline Age $50-70$ years & 19.65 & 17.12 & 2.509 & 21.80 & 11.91 & 9.477 & 3042 \\
\hline Age $>70$ years & 23.00 & 10.50 & 12.500 & 93.33 & 21.21 & 6.364 & 2 \\
\hline
\end{tabular}

As we sought to evaluate the precision of different diagnostic methods in breast cancer subgroups, we compared the correlations of ultrasound with histology, of mammography with histology and, respectively, of ultrasound with mammography.

Overall, the analyses comparing histology and ultrasound or histology and mammography showed no significant differences between the two non-invasive techniques $(p=0.18)$.

Both, ultrasound and mammography showed significantly higher correlations with histology in invasive ductal compared to invasive lobular cancers $(p=0.002$, $3.07 / p=0.008$ ).

Ultrasound and histology further showed a significantly better correlation for pT2 compared to pT1 cancers $(p=0.001)$. This correlation was also highly significantly superior for $\mathrm{pT} 2$ compared to $\mathrm{pT} 3$ cancers $(p=0.0002)$. Equivalent results could be detected in the correlation of mammography and histology, which was also significantly higher for $\mathrm{pT} 2$ compared to $\mathrm{pT} 1(p<0.001)$ or compared to pT3 $(p=0.026)$.

In the subgroup of invasive lobular cancers, histology showed a significantly higher correlation with mammography than with ultrasound $(p=0.01)$. There was no such difference in the invasive ductal cancer subgroup.

For HR negative cancers, ultrasound showed a significantly higher correlation with histology $(p<0.001)$. Size estimates by mammography were, however, significantly more accurate for $\mathrm{HR}$ positive than for $\mathrm{HR}$ negative
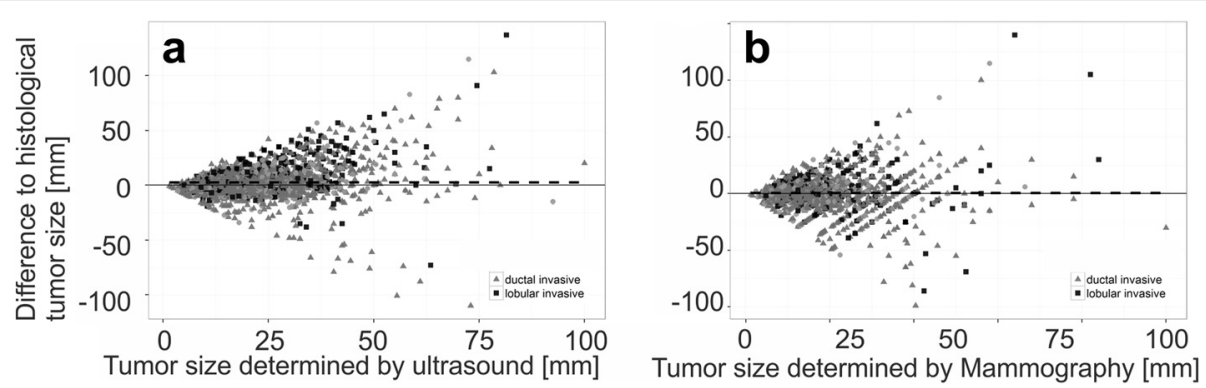

Fig. 1 Difference between sonographic, mammographic tumor size. Bland-Altman Diagrams of the Differences between tumor size as measured by ultrasound (a) and mammography (b) plotted against their respective mean value. Histological subtypes are indicated 
Table 2 Comparison of mammography and histology

\begin{tabular}{|c|c|c|c|c|c|c|c|}
\hline & $\begin{array}{l}\text { Mean } \\
\text { histologic } \\
\text { diameter }\end{array}$ & $\begin{array}{l}\text { Mean } \\
\text { mammographic } \\
\text { diameter }\end{array}$ & $\begin{array}{l}\text { Mean } \\
\text { difference }\end{array}$ & $\begin{array}{l}\text { Mean relative } \\
\text { difference } \\
\text { (\% mammographic } \\
\text { tumor diameter) }\end{array}$ & $\begin{array}{l}\text { SD Histologic } \\
\text { diameter }\end{array}$ & $\begin{array}{l}\text { SD mammographic } \\
\text { diameter }\end{array}$ & N \\
\hline Overall & 21.01 & 20.41 & 0.6012 & 14.85 & 12.5 & 11.91 & 4230 \\
\hline Endocrine non-responsive & 23.33 & 23.42 & -0.09015 & 11.08 & 13.603 & 13.431 & 599 \\
\hline Incomplete endocrine responsive & 20.45 & 20.05 & 0.39892 & 13.50 & 12.347 & 11.308 & 930 \\
\hline Highly endocine responsive & 20.70 & 19.87 & 0.82993 & 16.15 & 12.246 & 11.663 & 2693 \\
\hline Not applicable & 17.50 & 18.62 & -1.125 & 16.52 & 7.426 & 7.425 & 8 \\
\hline Ductal invasive & 20.59 & 20.30 & 0.2864 & 12.99 & 11.63 & 11.55 & 3132 \\
\hline Lobular invasive & 23.61 & 20.68 & 2.9325 & 31.22 & 15.97 & 13.32 & 474 \\
\hline G1 & 15.00 & 15.44 & -0.435 & 7.551 & 10.66 & 10.22 & 423 \\
\hline G2 & 20.16 & 19.65 & 0.5078 & 15.023 & 11.44 & 11.32 & 2623 \\
\hline G3 & 25.05 & 23.87 & 1.1782 & 17.078 & 14.02 & 12.78 & 1184 \\
\hline pT1 & 13.52 & 15.13 & -1.612 & 3.881 & 5.242 & 8.134 & 2408 \\
\hline pT2 & 28.51 & 26.11 & 2.399 & 24.427 & 6.995 & 10.127 & 1693 \\
\hline pT3 & 62.6 & 44.28 & 18.318 & 93.960 & 21.265 & 23.583 & 129 \\
\hline Age $<50$ years & 21.11 & 20.05 & 1.0606 & 16.54 & 12.54 & 11.53 & 808 \\
\hline Age $50-70$ years & 19.65 & 19.10 & 0.5579 & 16.12 & 12.18 & 11.49 & 2210 \\
\hline Age $>70$ years & 23.44 & 23.06 & 0.3757 & 11.44 & 12.67 & 12.47 & 1211 \\
\hline
\end{tabular}

non-responsive cancers, as evidenced by the superior correlation with histology $(p=0.0003)$.

Still, in both HR negative and HR positive cancers, mammography was inferior to ultrasound regarding the correlation with histology $(p<0.001 / p<0.001)$.

The correlation of mammography with histology was, however, significantly better for the HER2/neu negative than for the HER2/neu positive subgroup $(p<0.001)$. For the HER2/neu negative subgroup, mammography data showed a significantly higher correlation with histology whereas ultrasound was less precise $(p<0.001)$. In the HER2/neu positive subgroup, however, ultrasound came significantly closer to the histological size determination $(p=0.0001)$.

\section{Ultrasound tends to underestimate the tumor size in} invasive lobular cancers. Invasive lobular cancers showed a significantly higher percentage of grossly underestimated tumors (>35 mm difference to histology).

\section{Precision of ultrasound and mammography for $20 \mathrm{~mm}$ cutoff detection}

For further therapy, $20 \mathrm{~mm}$ tumor size is an important cutoff. We thus analyzed the sensitivity of mammography and ultrasound in detecting this tumor size cutoff. For detection of tumor sizes over $20 \mathrm{~mm}$, ultrasound was slightly more specific (0.752 versus 0.703$)$ and slightly more sensitive than mammography ( 0.824 versus 0.799). Ultrasound showed a higher cutoff detection rate
( 0.225 versus 0.172$)$, superior positive predictive $(0.555$ versus 0.424$)$ values. Mammography was superior only at negative predictive values $(0.919$ versus 0.927$)$.

\section{Patient age impacts both ultrasound and mammography precision}

The results in relation to patient age are shown in Table 4. As breast density decreases in older patients, we analyzed the results in different age groups. Patients aged $<50$ years, $50-70$ years and $>70$ years were compared respectively.

Higher patient age correlated with higher tumor size and respective $\mathrm{T}$ stage. Patients aged $<50$ years showed more HR negative cancers compared to older patients. The percentage of invasive ductal and lobular cancers was comparable in all age groups.

Both mammography and ultrasound were highly significantly superior for patients aged $>70$ years compared to patients aged $50-70$ years $(p<0.01)$. Both mammography and sonography achieved the lowest precision in patients aged $<50$ years compared to patients aged $50-$ 70 years $(p=0.024 / p=<0.001)$.

Still, the histology correlation of mammography and ultrasound did not significantly differ in any age group.

\section{Discussion}

In our study, the overall correlation between histology and mammography was 0.61 for mammography and 0.60 for ultrasound and thus did not show any significant 
Table 3 Correlation of tumor diameter in histology, Ultrasound and mammography

\begin{tabular}{|c|c|c|}
\hline Correlation overall & $\begin{array}{l}\text { Correlation } \\
\text { coefficient }\end{array}$ & N \\
\hline Histology - Ultrasound & 0.5981 & 5902 \\
\hline Histology - Mammography & 0.605 & 4230 \\
\hline Ultrasound - Mammography & 0.7369 & 3757 \\
\hline \multicolumn{3}{|c|}{ Correlation in ductal invasive cancer } \\
\hline Histology - Ultrasound & 0.6045 & 4257 \\
\hline Histology - Mammography & 0.6157 & 3132 \\
\hline Ultrasound - Mammography & 0.7319 & 2784 \\
\hline \multicolumn{3}{|c|}{ Correlation in lobular invasive cancer } \\
\hline Histology - Ultrasound & 0.5209 & 737 \\
\hline Histology - Mammography & 0.5271 & 474 \\
\hline Ultrasound - Mammography & 0.7012 & 424 \\
\hline \multicolumn{3}{|l|}{ Correlation for pT1 } \\
\hline Histology - Ultrasound & 0.3178 & 3450 \\
\hline Histology - Mammography & 0.2528 & 2408 \\
\hline Ultrasound - Mammography & 0.5922 & 2121 \\
\hline \multicolumn{3}{|l|}{ Correlation for pT2 } \\
\hline Histology - Ultrasound & 0.3938 & 2265 \\
\hline Histology - Mammography & 0.3864 & 1693 \\
\hline Ultrasound - Mammography & 0.6349 & 1534 \\
\hline \multicolumn{3}{|l|}{ Correlation for pT3 } \\
\hline Histology - Ultrasound & 0.1344 & 187 \\
\hline Histology - Mammography & 0.1989 & 129 \\
\hline Ultrasound - Mammography & 0.5527 & 102 \\
\hline
\end{tabular}

Correlation for endocrine non-responsive cancer ( $H R$ negative)

Histology - Ultrasound

Histology - Mammography

Ultrasound - Mammography

Correlation for incomplete endocrine responsive cancer (HR positive)

Histology - Ultrasound
Histology - Mammography
Ultrasound - Mammography

Correlation for highly endocrine responsive cancer (HR positive)

Histology - Ultrasound
Histology - Mammography
Ultrasound - Mammography
Correlation for HER2/neu positive cancer
Histology - Ultrasound
Histology - Mammography
Ultrasound - Mammography
Correlation for HER2/neu negative cancer
Histology - Ultrasound
$0.6124 \quad 3817$ $0.6334 \quad 2693$ $0.7407 \quad 2401$
Table 3 Correlation of tumor diameter in histology, Ultrasound and mammography (Continued)

\begin{tabular}{lll}
\hline Histology - Mammography & 0.6466 & 3278 \\
Ultrasound - Mammography & 00.7332 & 2956 \\
Correlation for patients aged <50 years & & \\
Histology - Ultrasound & 0.4658 & 1295 \\
Histology - Mammography & 0.5158 & 808 \\
Ultrasound - Mammography & 0.6922 & 729 \\
Correlation for patients aged 50-70 years & & \\
Histology - Ultrasound & 0.5970 & 3042 \\
Histology - Mammography & 0.5809 & 2210 \\
Ultrasound - Mammography & 0.7250 & 1941 \\
Correlation for patients aged >70 years & & \\
Histology - Ultrasound & 0.6700 & 1563 \\
Histology - Mammography & 0.6752 & 1211 \\
Ultrasound - Mammography & 0.7583 & 1086 \\
\hline Correlations with histology are shown for pairwise data, whereas correlations \\
between Ultrasound and mammography required complete datasets &
\end{tabular}

difference in terms of precision of tumor diameter measurement $(p=0.18)$. Both ultrasound and mammography did show a significantly higher correlation with histological tumor diameter in invasive ductal compared to invasive lobular cancers $(p=0.002 / p=0.008)$. For invasive lobular cancers, mammography turned out to be superior to ultrasound with respect to the correlation with histological tumor diameter $(p=0.01)$, whereas there was no advantage in the invasive ductal cancer subgroup. The analysis was focused on tumors detected by respective imaging. pT2 cancers could generally be assessed more precisely by both ultrasound and mammography whereas pT1 or pT3 showed more deviation. This result could be biased by the more accurate palpation of $\mathrm{T} 2$ tumors. While HR positive cancers did not show a difference between the precision of ultrasound and mammography, HR negative cancers show a highly significant advantage for ultrasound $(p<0.001)$. HER $2 /$ neu positive cancers also showed the superiority of ultrasound $(p<0.001)$ whereas mammography was superior in predicting the size of HER2/neu negative cancers $(p<0.001)$.

In line with Gruber et al. [5], we found ultrasound to underestimate histological tumor diameter. MRI data were not available for our study. Nevertheless, by comparing ultrasound and mammography data with histopathological findings, the precision of imaging-based tumor size determination could be assessed for the various biological subclasses of breast cancer. This showed that HR expression as well as HER2/neu overexpression impacts the precision achieved by imaging.

Hieken et al. [4] published that both ultrasound and mammography underestimated tumor size. In 180 cases 
Table 4 Patient age impacts both ultrasound and mammography precision

\begin{tabular}{|c|c|c|c|}
\hline Age (years) versus T stadium & pT1 & pT2 & pT3 \\
\hline$<50$ & 0.63591 & 0.33570 & 0.02839 \\
\hline $50-70$ & 0.63337 & 0.33754 & 0.02908 \\
\hline$>70$ & 0.46590 & 0.48902 & 0.04509 \\
\hline Age (years) versus Grading & G1 & G2 & G3 \\
\hline$<50$ & 0.095 & 0.530 & 0.375 \\
\hline $50-70$ & 0.122 & 0.627 & 0.251 \\
\hline$>70$ & 0.092 & 0.674 & 0.234 \\
\hline Age (years) versus HR expression & HR negative & HR incompletely responsive & HR positive \\
\hline$<50$ & 0.20 & 0.23 & 0.57 \\
\hline $50-70$ & 0.14 & 0.21 & 0.65 \\
\hline$>70$ & 0.10 & 0.21 & 0.69 \\
\hline Age (years) versus HER2/neu expression & HER2/neu negative & HER2/neu positive & \\
\hline$<50$ & 0.82 & 0.18 & \\
\hline $50-70$ & 0.85 & 0.15 & \\
\hline$>70$ & 0.88 & 0.12 & \\
\hline Age (years) versus histological subtype & Ductal invasive & Lobular invasive & \\
\hline$<50$ & 0.75 & 0.093 & \\
\hline $50-70$ & 0.72 & 0.133 & \\
\hline$>70$ & 0.71 & 0.128 & \\
\hline Age (years) & HER2/neu negative & HER2/neu positive & \\
\hline$<50$ & 0.82 & 0.18 & \\
\hline $50-70$ & 0.85 & 0.15 & \\
\hline$>70$ & 0.88 & 0.12 & \\
\hline
\end{tabular}

Relative Quantifications for T stadium, Grading, HR expression, HER2/neu expression or histological subtype in relation to age are shown

of invasive breast cancers, they found ultrasound to be more accurate. In clear contrast to their results, we could show distinct differences of imaging precision in invasive ductal and invasive lobular cancers and thus provide evidence for the importance of biological cancer subgroups for imaging.

Dummin and colleagues [3] found, that ultrasound underestimates breast cancer size. Mammography turned out to be the most precise tool for predicting histological tumor size. However, they did not compare different biological cancer subgroups regarding the correlations between histological, sonographic and mammographic tumor diameter.

It has to be considered that our retrospective study is an analysis of longitudinal study data. Further studies should investigate not only the maximum tumor diameter but for example three-dimensional tumor size. Improved ultrasound technologies such as 3D ultrasound make this possible. Our analysis is based on a large set of patient data, even though ultrasound and mammography data were not available for all patients. Furthermore, there was no information about breast density in imaging according to the American college of radiology (ACR). A great advantage of the longitudinal BRENDA I study is that the data were collected under realistic daily routine conditions. Precise data also exist for exact histological tumor diameter and all histological subtypes of breast cancer are represented. We could thus show that both ultrasound and mammography are reasonably precise in assessing tumor size. Mammography seems favorable for HER2/neu negative and invasive lobular cancers. Ultrasound is more precise for HER2/neu positive and HR negative invasive ductal cancers.

\section{Conclusion}

We provide evidence that the prediction of tumor size by ultrasound and mammography in breast cancer is reliable in this large multicentered daily routine cohort of primary breast cancer patients. Nevertheless, our data suggest that inherent features of individual tumor subgroups influence the non-invasive assessment of tumor size. Taking this into consideration may further improve the interpretation of imaging data for therapeutic decisions. 


\section{Abbreviations}

ACR: American College of Radiology; CLIS / LIN: carcinoma lobulare in situ; DCIS: ductal carcinoma in situ; Fig.: figure; HR: hormone receptor (Estrogen- and Progesterone-Receptor); MRI: magnetic resonance imaging; MRM: modified radical mastectomy; NOS: not otherwise specified breast cancer; NST: no special type breast cancer; SD: standard deviation

\section{Funding}

BMBF (Bundesministerium für Bildung und Forschung, Germany) Grant $01 Z$ P0505

\section{Availability of data and materials}

The analyzed data and materials can be obtained by the authors upon request.

\section{Authors' contributions}

Data Analysis and Manuscript Design: RS, LS. Corrections of Paper and Project Design: JD, TS, JW, CB, MK. Statistical Analysis: MB, DW, MW. Conception and Study Design: RK, WJ, AW. All authors have read and approved the manuscript.

\section{Authors' information}

Not applicable.

\section{Competing interests}

The authors declare that they have no competing interests.

\section{Consent for publication}

Not applicable.

\section{Ethics approval and consent to participate}

The study was approved by the Ulm University Ethics committee. Patients gave informed consent to participate.

\section{Author details}

${ }^{1}$ Department of Obstetrics and Gynecology, Würzburg University Hospital, Josef-Schneider-Str. 4, 97080 Würzburg, Germany. ${ }^{2}$ Insititute of Medical Biostatistics, Epidemiology and Informatics (IMBEI), Mainz University Hospital, Mainz, Germany. ${ }^{3}$ Department of Obstetrics and Gynecology, Ulm University Hospital, Ulm, Germany. ${ }^{4}$ e-Science, Bremen University, Bremen, Germany.

Received: 25 January 2016 Accepted: 20 June 2016

Published online: 13 July 2016

\section{References}

1. Perou CM, Sorlie T, Eisen MB, van de Rijn M, Jeffrey SS, Rees CA, et al. Molecular portraits of human breast tumours. Nature. 2000;406(6797):747-52.

2. Vijver MJ. WHO classification of tumours of the breast - IARC WHO classification of tumours, No 4. 4th ed. Lyon: International Agency for Research on Cancer; 2012.

3. Dummin LJ, Cox M, Plant L. Prediction of breast tumor size by mammography and sonography - A breast screening experience. Breast. 2007;16(1):38-46.

4. Hieken TJ, Harrison J, Herreros J, Velasco JM. Correlating sonography, mammography and pathology in the assessment of breast cancer size. Am J Surg. 2001;182(4):351-4

5. Gruber IV, Rueckert M, Kagan KO, Staebler A, Siegmann KC, Hartkopf A, et al. Measurement of tumour size with mammography, sonography and magnetic resonance imaging as compared to histological tumour size in primary breast cancer. BMC Cancer. 2013;13:328.

6. Bosch AM, Kessels AG, Beets GL, Rupa JD, Koster D, van Engelshoven JM et al. Preoperative estimation of the pathological breast tumour size by physical examination, mammography and ultrasound: a prospective study on 105 invasice tumours. Eur J Radiol. 2003:48(3):285-92.

7. Snelling JD, Abdullah N, Brown G, King DM, Moskovic E, Gui GP. Measurement of tumour size in case selection for breast cancer therapy by clinical assessment and ultrasound. Eur J Surg Oncol. 2004;30(1):5-9.

8. Ramirez SI, Scholle M, Buckmaster J, Paley RH, Knowdley GC. Breast cancer tumor size assessment with mammography, ultrasound, and magnetic resonance imaging at a community based multidisciplinary breast center. Am Surg. 2012;78(4):440-6.
9. Kreienberg RAU, Follmann M, Kopp I, Kühn T, Wöckel A, Zemmler T. Diagnosis and Treatment of Patients with Primary and Metastatic Breast Cancer. Interdisciplinary S3 Guideline. Berlin: German Cancer Socienty; 2012. http://www.leitlinienprogramm-onkologie.org.

10. Nothacker M, Duda V, Hahn M, Warm M, Degenhardt F, Madjar H, et al. Early detection of breast cancer: benefits and risk of supplemental breast ultrasound in asymptomatic women with mammographically dense breast tissue. A systematic review. BMC Cancer. 2009;9:335.

11. (NICE) NNIfCE. Early and locally advanced breast cancer: diagnosis and treatement. 2009

12. Shoma A, Mouramed A, Ameen M, Abdelwahab A. Ultrasound for accurate measurement of invasice breast cancer tumor size. Breast J. 2006;12(3):252-6.

13. Houssami N, Turner R, Morrow M. Preoperative magnetic resonance imaging in breast cancer: meta-analysis of surgical outcomes. Ann Surg. 2013:257(2):249-55.

14. Houssami N, Ciatto S, Macaskill P, Lord SJ, Warren RM, Dixon JM, et al. Accuracy and surgical impact of magnetic resonance imaging in breast cancer staging: Systematic review and meta-analysis in detection of multifocal and multicentric cancer. J Clin Oncol. 2008;26(19):3248-58.

15. Yi A, Cho N, Yang KS, Han W, Noh DY, Moon WK. Breast cancer recurrence in patients with newly diagnosed breast cancer without and with preoperative MR imaging: a matched cohort study. Radiology. 2015:276(3): 695-705.doi:10.1148/radiol.2015142101

16. Goldhirsch A, Wood WK, Gelber RD, Coates AS, Thurlimann B, Senn HJ. Progress and promise: highlights of the international expert consensus on the primary therapy of early breast cancer 2007. Ann Oncol. 2007:18(7):1133-44.

17. Team RC: A Language and Environment for Statistical Computing. http:// www.R-project.org; R Foundation for Statistical Computing. Vienna, Austria, 20142015.

\section{Submit your next manuscript to BioMed Central and we will help you at every step:}

- We accept pre-submission inquiries

- Our selector tool helps you to find the most relevant journal

- We provide round the clock customer support

- Convenient online submission

- Thorough peer review

- Inclusion in PubMed and all major indexing services

- Maximum visibility for your research

Submit your manuscript at www.biomedcentral.com/submit 